\title{
Article \\ Improving Serial Detection Using MAP Algorithm for Bit-Patterned Media Recording
}

\author{
Thien An Nguyen (D) and Jaejin Lee *(D)
}

Department of Information Communication Convergence Technology, Soongsil University, Seoul 06978, Korea; anthienng1995@soongsil.ac.kr

* Correspondence: zlee@ssu.ac.kr; Tel.: +82-2-820-0901

Citation: Nguyen, T.A.; Lee, J. Improving Serial Detection Using MAP Algorithm for Bit-Patterned Media Recording. Appl. Sci. 2022, 12, 1979. https://doi.org/10.3390/ app12041979

Academic Editor: David Megías

Received: 21 December 2021

Accepted: 12 February 2022

Published: 14 February 2022

Publisher's Note: MDPI stays neutral with regard to jurisdictional claims in published maps and institutional affiliations.

Copyright: (C) 2022 by the authors. Licensee MDPI, Basel, Switzerland. This article is an open access article distributed under the terms and conditions of the Creative Commons Attribution (CC BY) license (https:// creativecommons.org/licenses/by/ $4.0 /)$.

\begin{abstract}
Bit-patterned media recording (BPMR) is a promising technology for increasing the areal density (AD) of magnetic data storage systems. In BPMR, the non-magnetic zone is reduced to increase $\mathrm{AD}$, which causes serious problems such as two-dimensional (2D) interference and track mis-registration (TMR). Therefore, in previous studies, a serial detection, which exploits the Viterbi algorithm (VA), was proposed to combat 2D interference. Because the VA lacks prior information from the inner detector to the outer detector, in this study, we use the maximum a posteriori (MAP) algorithm to supply prior information from the inner detector to the outer detector. Simulation results show that the proposed model can improve the bit error ratio (BER) performance of serial detection, especially in cases where the BPMR suffers from TMR effects.
\end{abstract}

Keywords: bit-patterned media recording; detection; interference; maximum a posteriori (MAP)

\section{Introduction}

Bit-patterned media recording (BPMR) helps in overcoming the limitations of the areal density (AD) of $1 \mathrm{~Tb} / \mathrm{in}^{2}$ due to superparamagnetism in current magnetic hard disc drive systems. In BPMR, the magnetic islands are surrounded by a non-magnetic zone, and the data are magnetized and stored on the magnetic islands. To increase the AD, the non-magnetic zone must be reduced to bring the magnetic islands closer together. Thus, two-dimensional (2D) interference, which includes the inter-track interference (ITI) from the cross-track direction and the inter-symbol interference (ISI) from the down-track direction, is important in BPMR systems. In addition, BPMR suffers from track misregistration (TMR), media noise, and so on [1,2]. In addition, two-dimensional (2D) magnetic recording (TDMR) [3,4] and heat-assisted magnetic recording (HAMR) [5] also are new technologies proposed to combat the superparamagnetism and researched simultaneously with the BPMR.

To handle 2D interference, we can use certain signal processing techniques, such as error-correcting modulation codes and good detection algorithms. By using the errorcorrecting modulation code, BPMR systems can achieve a higher performance by sacrificing a part of the storage system to store the parity data. Thus, we use the detection algorithm to avoid interference and loss of data storage space. To combat ITI with low implementation cost, Sadeghian and Barry proposed an effective detection technique in [6]. In addition, Shi and Barry [7] also proposed a multitrack detector with 2D pattern-dependent noise prediction, which significantly outperforms the conventional 2D Viterbi detector when the channel noise is pattern-dependent. Cideciyan et al. proposed a partial response maximum likelihood (PRML) method to equalize the received signal to obtain the desired signal which only suffer interference from the target [8]. Nabavi and Kumar proposed a generalized partial response (GPR) target based on PRML [9]. This helped in the development of several detection algorithms. Serial detection for the BPMR systems based on the coefficient estimation was also introduced using the GPR target [10,11]. Serial detection is inspired 
by the 2D soft-output Viterbi algorithm (SOVA) for BPMR systems [12]. The 2D SOVA is composed of two parallel 1D SOVAs. One SOVA is used for horizontal detection, and the other is used for vertical detection. Because serial detection is implemented via the Viterbi algorithm (VA), the detection lacks prior information from the inner detector to the outer detector. In the previous studies, serial detection using VA keeps the information of a candidate symbol and ignores the other symbols. The information of these symbols is called the prior information. Therefore, in this study, to solve the above-mentioned problem, we use the maximum a posteriori probability (MAP) algorithm to preserve the information of the ignored symbols to the final decision part (Log Detection).

In the proposed model, the output of the inner detection was split into two branches. One was supplied to the outer detection to achieve a soft output. The other was combined with the soft output of the outer detection to detect the original signal using log detection. The results of the simulation show that the proposed model can improve the performance of serial detection by exploiting the MAP.

The remainder of this paper is organized as follows. In Section 2, we present the proposed model. In Section 3, we present the simulation results and discussion. In Section 4, the conclusions are presented.

\section{Proposed Model}

\subsection{Horizontal Detection and Vertical Detection}

Figure 1 shows the proposed detection model. The received signal $y[j, k]$ passes through equalizer $\mathbf{F}$, and the equalizer output signal $z[j, k]$ is an approximation of the desired signal $d[j, k]$. In other words, the horizontal detection receives the signal $z[j, k]$, which is corrupted by the $2 \mathrm{D}$ channel interference and approximated to the desired signal by the GPR target G. In [10], the authors analyzed the 2D interference in target $\mathbf{G}$ into two serial 1D interferences. Because the output of the inner detection (horizontal detection) is a 6-level signal $\{-1-2 p$, $-1,-1+2 p, 1-2 p, 1,1+2 p\}$, the trellis of the horizontal detection includes 36 states and six input branches in each state, as shown in Figure 2. We denote these six values as the $R_{0}, R_{1}, \ldots, R_{5}\left(R_{0}=-1-2 p, R_{1}=-1, R_{2}=-1+2 p, R_{3}=1-2 p, R_{4}=1\right.$, and $\left.R_{5}=1+2 p\right)$ for convenience.

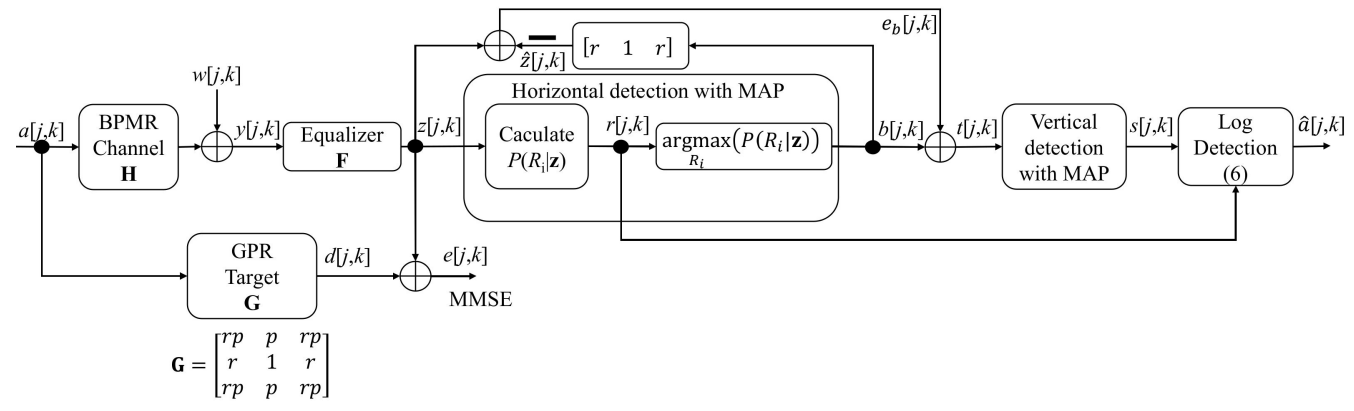

Figure 1. Proposed detection model. 


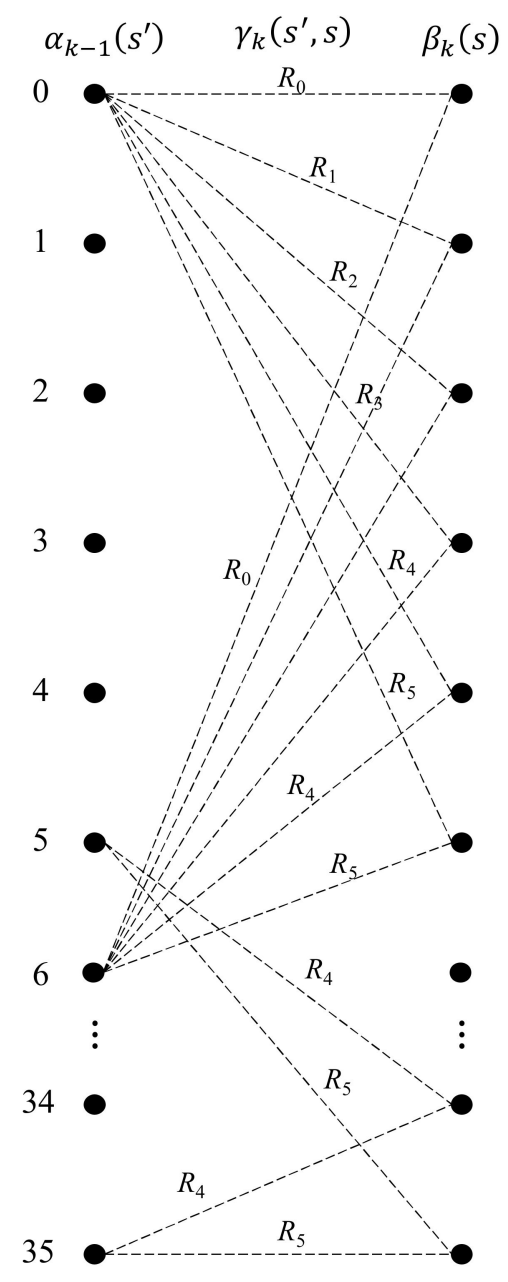

Figure 2. Proposed detection model.

In [13], the authors proposed $P\left(u_{k}=+1 \mid \mathbf{y}\right)$ and $P\left(u_{k}=-1 \mid \mathbf{y}\right)$, where $u_{k}$ is the output of the MAP detection at time $k$, and $\mathbf{y}$ is the received symbol of MAP detection. However, in our proposed model, the output of the MAP has more than two states. Therefore, we simply calculate the conditional probability $P\left(R_{i} \mid \mathbf{z}\right)$ as follows:

$$
\begin{aligned}
& P\left(R_{i} \mid \mathbf{z}\right)= \sum_{R_{i}} P(s \prime, s \mid \mathbf{z})=\sum_{R_{i}} \frac{P(s \prime, s, \mathbf{z})}{P(\mathbf{z})} \\
&= \sum_{R_{i}} \frac{P\left(s \prime, \mathbf{z}_{<k}\right) P\left(\mathbf{z}_{k}, s \mid s^{\prime}\right) P\left(\mathbf{z}_{>k} \mid s\right)}{P(\mathbf{z})} \\
&=\sum_{R_{i}} \frac{\alpha_{k-1}(s \prime) \gamma_{k}(s \prime, s) \beta_{k}(s)}{P(\mathbf{z})} \\
& \text { with } i=0,1, \ldots, 5
\end{aligned}
$$

where $s /$ is the previous state, $s$ is the current state, $\mathbf{z}$ is the received symbol of the MAP detection in the proposed model, $\alpha_{k-1}(s \prime)=P\left(s /, \mathbf{z}_{<k}\right)$ is the joint probability of the previous state $s /$ and the sequence received in the past received sequence $\mathbf{z}_{<k}, \gamma_{k}\left(s^{\prime}, s\right)=P\left(\mathbf{z}_{k}, s \mid s^{\prime}\right)$ represents the conditional probability of the current state $s$ and the current received symbol $\mathbf{z}_{k}$ given the previous state $s /$, and $\beta_{k}(s)=P\left(\mathbf{z}_{>k} \mid s\right)$ is the conditional probability of the future received sequence $\mathbf{z}_{>k}$ under the current state $s$. 
By using Equation (1), we can calculate $P\left(R_{i} \mid \mathbf{z}\right)$ for $R_{0}, R_{1}, \ldots, R_{5}$. Let $r[j, k]=\left[P\left(R_{0} \mid \mathbf{z}\right)\right.$, $P\left(R_{1} \mid \mathbf{z}\right), P\left(R_{2} \mid \mathbf{z}\right), P\left(R_{3} \mid \mathbf{z}\right), P\left(R_{4} \mid \mathbf{z}\right)$, and $\left.P\left(R_{5} \mid \mathbf{z}\right)\right]$. Then, we find the maximum $P\left(R_{i} \mid \mathbf{z}\right)$ in $r[j, k]$. In other words, we have

$$
b[j, k]=\underset{R_{i}}{\operatorname{argmax}}\left(P\left(R_{i} \mid \mathbf{z}\right)\right) .
$$

With Equation (2), the signal $b[j, k]$ is a hard decision value. To create a soft output for the signal $b[j, k]$, we applied the feedback in [11]. The signal $b[j, k]$ passes through the interference target $\left[\begin{array}{lll}r & 1 & r\end{array}\right]$ to create the estimated signal $\hat{z}[j, k]$. The difference of $z[j, k]$ and $\hat{z}[j, k]$ is the estimated noise $e_{b}[j, k]$. Finally, the soft output of signal $b[j, k]$ is given as follows:

$$
t[j, k]=b[j, k]+e_{b}[j, k] .
$$

To detect the signal $t[j, k]$ in Equation (3), we used vertical detection with the Bahl, Cocke, Jelinek, and Raviv (BCJR) algorithm in [13]. Thus, the outputs of the vertical detection $s[j, k]$ are the conditional probabilities $P\left(s_{k}=+1 \mid \mathbf{t}\right)$ and $P\left(s_{k}=-1 \mid \mathbf{t}\right)$ for the signals " 1 " and " -1 ", respectively. We used the signal $r[j, k]$ and $s[j, k]$ for log detection as follows:

$$
\begin{gathered}
\operatorname{num}[j, k]=P\left(s_{k}=1 \mid \mathbf{x}\right) \sum_{i} P\left(R_{i} \mid \mathbf{z}\right) \\
\text { with } i=1,4,2,5 . \\
\operatorname{den}[j, k]=P\left(s_{k}=-1 \mid \mathbf{x}\right) \sum_{i} P\left(R_{i} \mid \mathbf{z}\right) \\
\text { with } i=0,3,1,4 . \\
\hat{a}[j, k]=\log \left(\frac{n u m[j, k]}{\operatorname{den}[j, k]}\right)
\end{gathered}
$$

To obtain Equations (4) and (6), we consider the relationship between signals $s[j, k]$ and symbol $R_{i}$ listed in Table 1 .

Table 1. Dependence of the signal $s[j, k]$ and symbols $R_{i}$.

\begin{tabular}{ccccc}
\hline Symbol & Value & $s[j-\mathbf{2}, \boldsymbol{k}]$ & $s[j-\mathbf{1}, \boldsymbol{k}]$ & $s[j, \boldsymbol{k}]$ \\
\hline$R_{0}$ & $-1-2 p$ & -1 & -1 & -1 \\
$R_{1}$ & -1 & -1 & -1 & 1 \\
$R_{3}$ & $1-2 p$ & -1 & 1 & -1 \\
$R_{4}$ & 1 & -1 & 1 & 1 \\
$R_{1}$ & -1 & 1 & -1 & -1 \\
$R_{2}$ & $-1+2 p$ & 1 & -1 & -1 \\
$R_{4}$ & 1 & 1 & 1 & 1 \\
$R_{5}$ & $1+2 p$ & 1 & 1 & \\
\hline
\end{tabular}

The values of the signals $[j, k]$ depend on the values of signal $r[j, k]$. The probabilities that depend on the signal $s_{k}=1$ are the four cases of $r[j, k]$ such as $\{-1,1,-1+2 p, 1+2 p\}$. Thus, we obtained the relation in Equation (4). Similarly, the probabilities depending on the signal $s_{k}=-1$ are the other four cases of $\left(R_{0}=-1-2 p, R_{3}=1-2 p, R_{1}=-1\right.$, $\left.R_{4}=1\right)$. Thus, the relation in Equation (5) was obtained. Generally, in "Log Detection", the likelihood information from the vertical and horizontal detection is as the likelihood information and the prior information, respectively, of the original signal $a[j, k]$. Therefore, using Equation (6), we calculated the a posteriori log-likelihood ratio (LLR) of the signal $\hat{a}[j, k]$. Finally, we demodulated the signal $\hat{a}[j, k]$ into the original $\hat{u}[k]$.

\subsection{Improving Serial Detection with MAP Algorithm}

After the received signal $y[j, k]$ goes through the equalizer, the signal $z[j, k]$ is similar to the signal influenced by the vertical interference $[p 1 p]^{T}$ and the horizontal interference $[r 1$ r]. This is presented in Figure 3. 


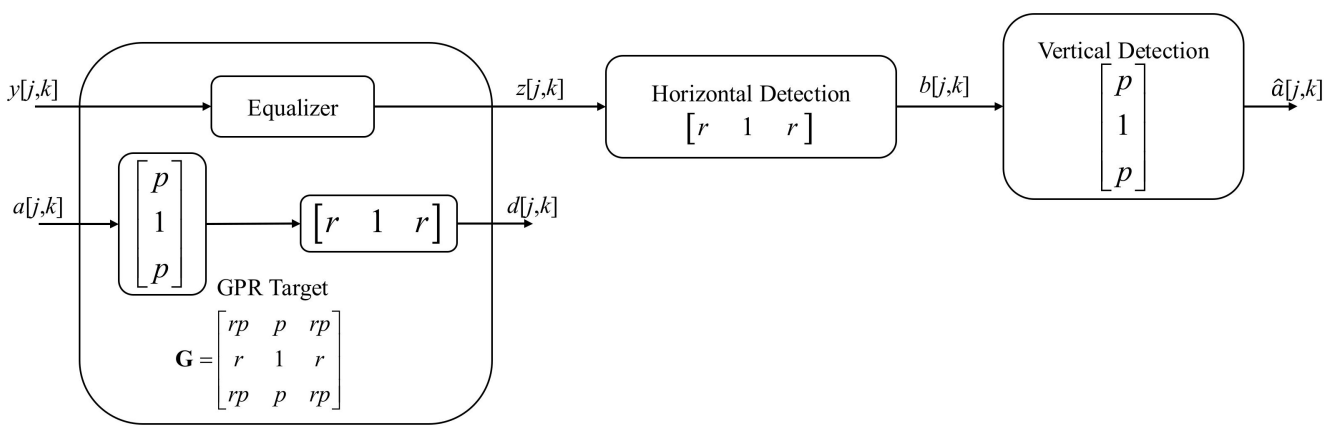

Figure 3. Serial detection model.

After detecting $z[j, k]$ by horizontal detection, we achieve the signal $b[j, k]$. Depending on how to decide the values of $b[j, k]$, we can discriminate three cases, such as hard output serial detection [10], soft output serial detection [11], and our proposed model. This is presented in Figure 4.

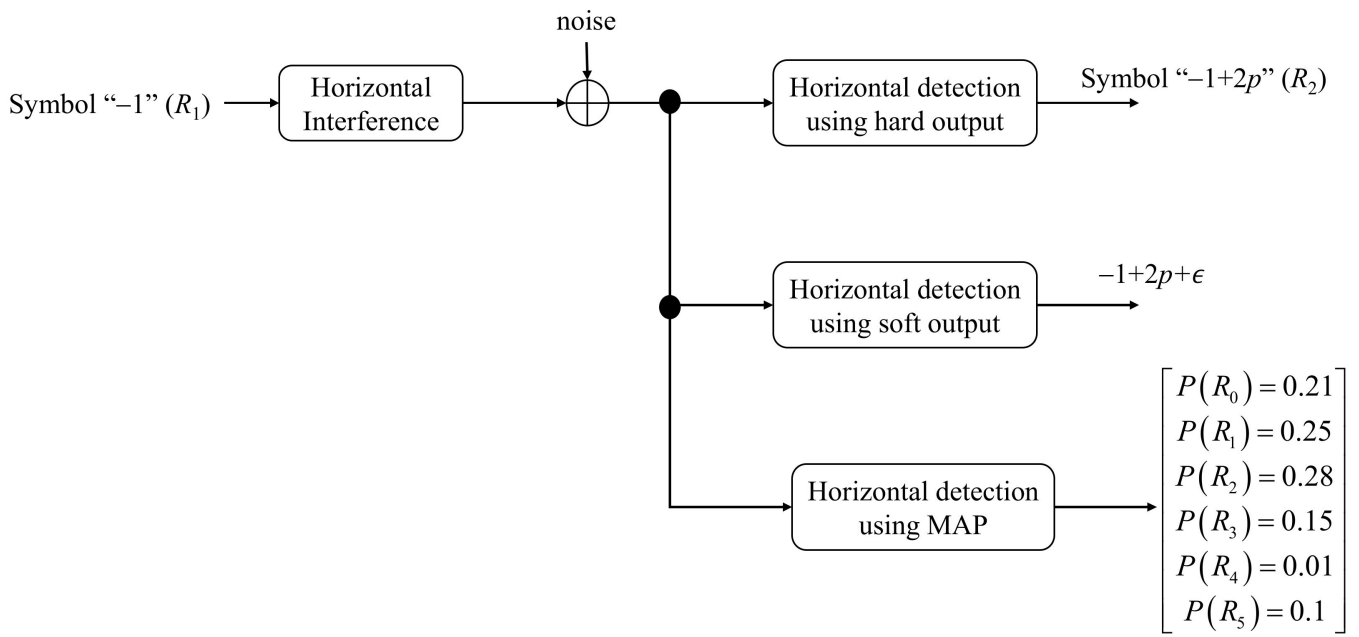

Figure 4. Effect of MAP algorithm on the output of horizontal detection.

In Figure 4, we can see that the probability of $R_{2}$ in our proposed model has the largest value (0.28). We can choose $R_{2}$ for applying the method in [11] and create a soft output similar to [11]. This soft output is supplied to the vertical detection. Here, the vertical detection with MAP algorithm estimates the probability of " -1 " and " 1 " in the modulated signal $a[j, k]$. This is the second effect of the MAP algorithm on serial detection. This is present in Figure 5.

Now, we have the probability of the symbols from the output of horizontal detection and the probability of -1 and 1 from the output of vertical detection. Therefore, we consider the relationship between symbol $\left\{R_{0}, R_{1}, \ldots, R_{5}\right\}$ and data $\{-1,1\}$ in the modulated signal $a[j, k]$ such as the one in Table 1 . From Table 1 , we can see that if $s[j, k]$ is -1 , the output of horizontal detection can achieve the symbols $R_{0}, R_{3}, R_{1}$, and $R_{4}$. If $s[j, k]$ is 1 , the output of horizontal detection can achieve the symbols $R_{1}, R_{4}, R_{2}$, and $R_{5}$. Therefore, "Log Detection" applies Equations (4)-(6) to decide the values in $a[j, k]$.

With "Log Detection," it shows the final effect of the MAP algorithm on the serial detection. We use the MAP algorithm to provide more finely tuned information from horizontal detection to vertical detection. This combination creates the soft decision for all processing in serial detection and avoids the quantization error. 


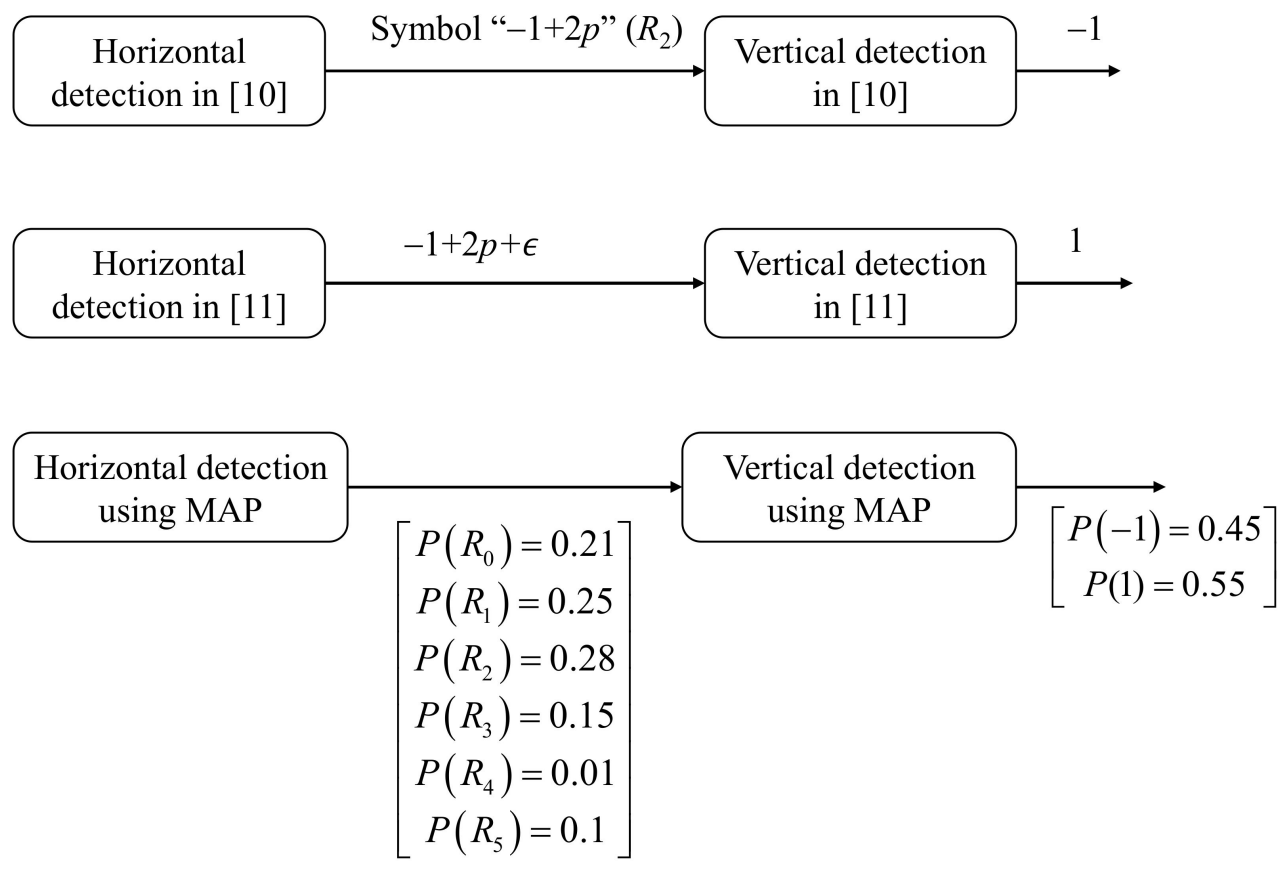

Figure 5. Effect of MAP algorithm on the output of vertical detection.

\section{Simulations}

\subsection{BPMR Channel}

In Figure 1, for simplification, we did not include the modulation and demodulation blocks. However, in the simulation, we implemented the modulation of the original signal $u[k] \in\{0,1\}$ into the signal $a[j, k] \in\{-1,+1\}$ and the demodulation of signal $\hat{a}[j, k]$ into the estimated original signal $\hat{u}[k]$.

The received signal $y[j, k]$ is expressed as follows:

$$
y[j, k]=a[j, k] * h[j, k]+w[j, k],
$$

where $j$ and $k$ are the discrete indices in the down- and cross-track directions, respectively; $w[j, k]$ is additive white Gaussian noise (AWGN) with zero mean, variance $\sigma^{2}$. The BPMR channel pulse response $h[j, k]$ is expressed as follows:

$$
h[j, k]=P\left(j T_{x}, k T_{q}-\Delta_{o f f}\right),
$$

where $P(x, q)$ is expressed as follows [14]:

$$
P(x, q)=A \exp \left(-\frac{1}{2 c^{2}}\left[\left(\frac{x+\Delta_{x}}{P W_{x}}\right)^{2}+\left(\frac{q+\Delta_{q}}{P W_{q}}\right)^{2}\right]\right),
$$

where $x$ and $q$ are the down- and cross-track directions, respectively; $\Delta_{x}$ and $\Delta_{q}$ are the down- and cross-track bit location fluctuations, respectively; $A=1$. The constant, $c=1 / 2.3548$ [15], and $P W_{x}$ and $P W_{q}$ are the $P W_{50}$ components of the down- and crosstrack, respectively. TMR is defined as the ratio between the head offset size and the magnetic island period, and is expressed as follows:

$$
\operatorname{TMR}(\%)=\frac{\Delta_{o f f}}{T_{q}} .
$$

We created a BPMR channel with an areal density of $3 \mathrm{~Tb} / \mathrm{in}^{2}\left(0.465 \mathrm{~Tb} / \mathrm{cm}^{2}\right)$ $\left(T_{x}=T_{q}=14.5 \mathrm{~nm}\right)$ [16]. The signal-to-noise ratio $(\mathrm{SNR})$ was defined as SNR $=10 \log _{10}\left(1 / \sigma^{2}\right)$. 
To simplify, we first applied our proposed model to the BPMR channel without the TMR effect $\left(\Delta_{o f f}=0\right)$. This channel has the following parameters:

$$
\mathbf{H}=\left[\begin{array}{ccc}
0.0824 & 0.3876 & 0.0824 \\
0.2125 & 1 & 0.2125 \\
0.0824 & 0.3876 & 0.0824
\end{array}\right]
$$

\subsection{Results and Discussions}

Figure 6 shows the comparison between the bit error ratio (BER) performance of the proposed model and the serial detection methods in $[10,11]$. The proposed model outperforms serial detection method at $14 \mathrm{~dB}$. The proposed model can achieve a gain of approximately $0.5 \mathrm{~dB}$ and $1.5 \mathrm{~dB}$ compared to the serial soft output [10] and serial hard output, respectively [11], at a BER of $3 \times 10^{-5}$.

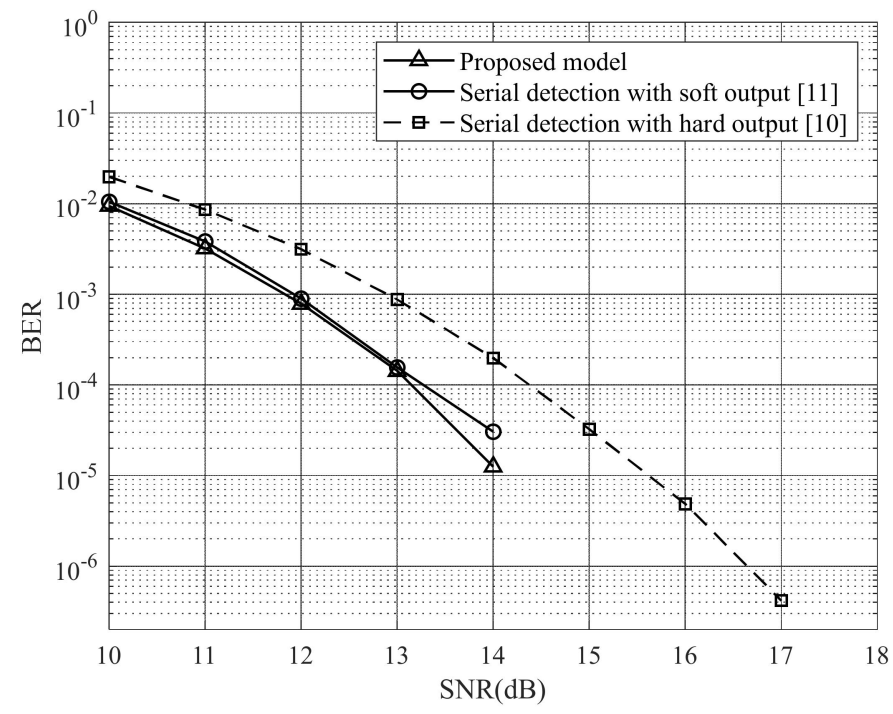

Figure 6. BER performance of the proposed model.

The BER performance of the proposed model in the BPMR channel with a 10\% TMR effect is shown in Figure 7.

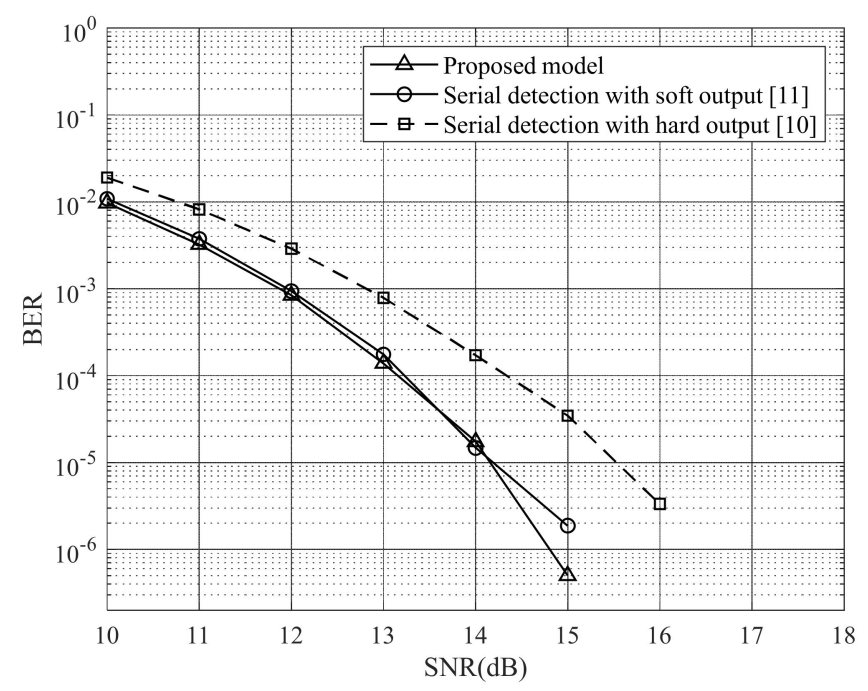

Figure 7. BER performance of the proposed model with 10\% TMR. 
The BER performance of the proposed model did not change compared to that of the channel without TMR when the level of the TMR was $10 \%$. In other words, the proposed model could resist $10 \%$ TMR.

Next, we varied the level of the TMR effect from $10 \%$ to $30 \%$ at $15 \mathrm{~dB}$ to evaluate the ability of the proposed model to resist the effect of TMR. Figure 8 shows the variation in BER performance with the change in TMR effect. The proposed model shows a better performance than serial detection with VA [11] up to $30 \%$ TMR.

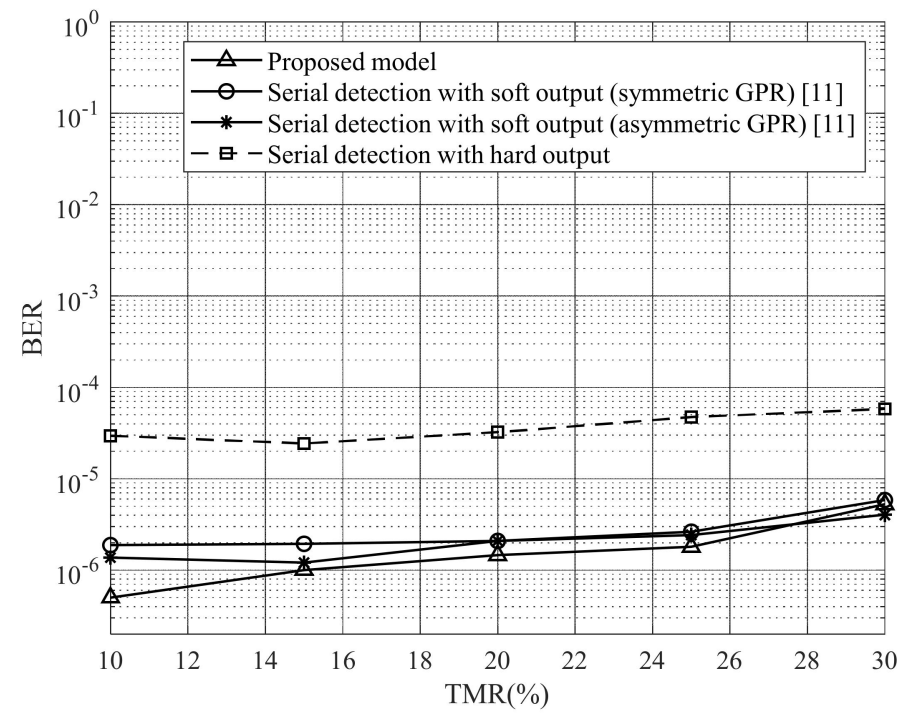

Figure 8. BER performance of the proposed model according to TMR at SNR $=15 \mathrm{~dB}$.

We implement the simulation with the position fluctuation of $6 \%$ and compare it to the serial soft-output detection in [11]. The results are presented in Figure 9. We can see that our proposed model still can improve the BER performance. Figure 10 shows the BER performance of the proposed model when we vary the position fluctuation from $6 \%$ to $18 \%$ at $15 \mathrm{~dB}$ to present the BER expression as the function of the position fluctuation. The proposed model performs better than other schemes even though they converge when the position fluctuation increases.

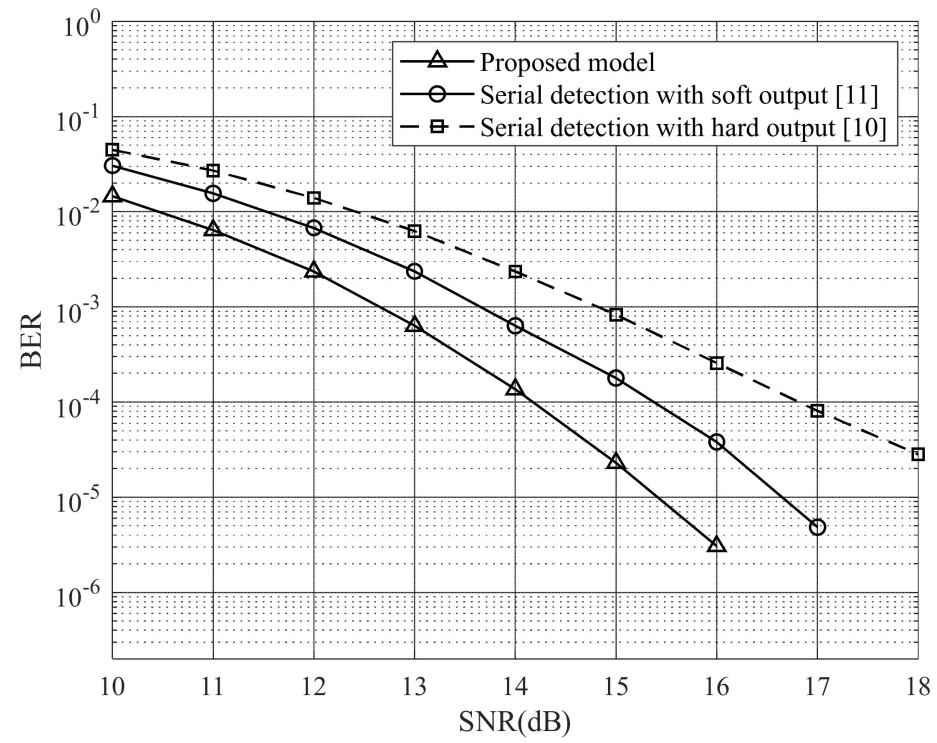

Figure 9. BER performance of the proposed model for $6 \%$ position fluctuation. 


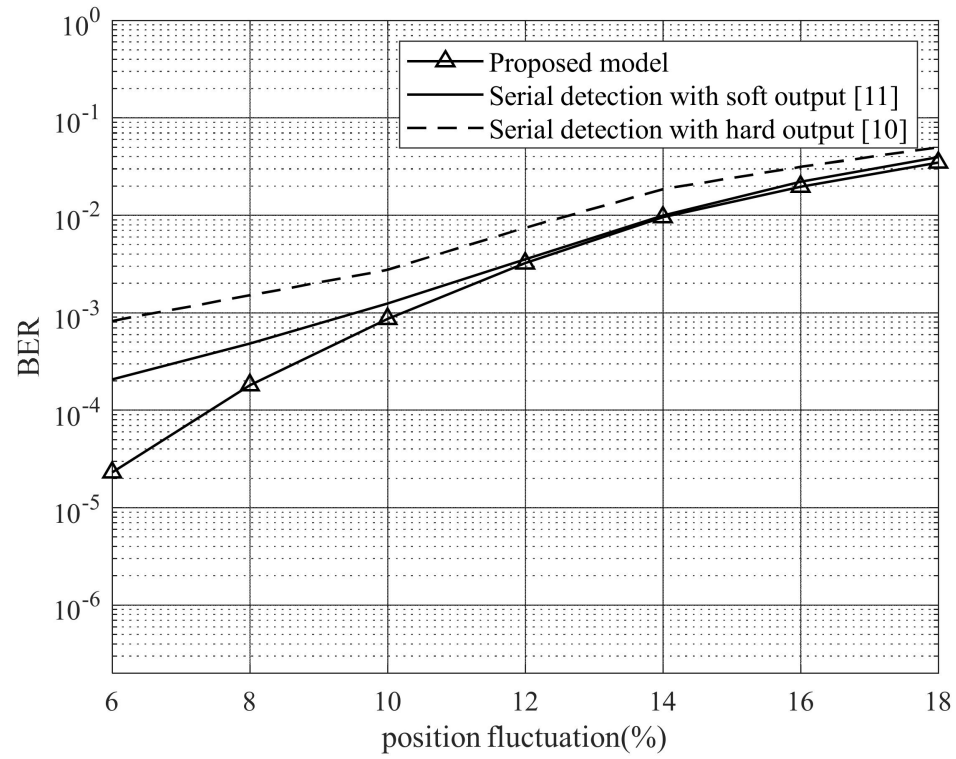

Figure 10. BER performance of the proposed model according to position fluctuation at $\mathrm{SNR}=15 \mathrm{~dB}$.

\section{Conclusions}

We proposed a serial detection method using the MAP algorithm. In this model, we modified the MAP algorithm to be suitable for the 6-level signal of the inner detector in the serial detection. Moreover, we used the probability information of the output symbols from the inner detection for supplying the output of the outer detector. In other words, we preserved the prior information from the inner detector to the outer detector. This helped our proposed model to improve the BER performance of serial detection. In addition, the results of the simulation also showed that our proposed model could resist the TMR effect by up to $30 \%$. Finally, our proposed model can still improve the BER performance in the presence of up to $12 \%$ position fluctuation.

Author Contributions: Conceptualization, T.A.N. and J.L.; methodology, T.A.N. and J.L.; software, T.A.N.; validation, T.A.N. and J.L.; formal analysis, T.A.N.; investigation, T.A.N. and J.L.; writingoriginal draft preparation, T.A.N.; writing—review and editing, T.A.N. and J.L.; supervision, J.L.; project administration, J.L.; funding acquisition, J.L. All authors have read and agreed to the published version of the manuscript.

Funding: This work was supported by the National Research Foundation of Korea (NRF) grant funded by the Korea government. (MSIT) (2021R1A2C1011154).

Conflicts of Interest: The authors declare no conflict of interest.

\section{References}

1. Chang, W.; Cruz, J.R. Inter-track interference mitigation for bit-patterned magnetic recording. IEEE Trans. Magn. 2010, 46, 3899-3908. [CrossRef]

2. Nabavi, S.; Kumar, B.V.K.V.; Bain, J.A. Two-dimensional pulse response and media noise modeling for bit-patterned media. IEEE Trans. Magn. 2008, 44, 3789-3792. [CrossRef]

3. Thompson, D.A.; Best, J.S. The future of magnetic data storage technology. IBM J. Res. Dev. 2000, 44, 311-322. [CrossRef]

4. Garani, S.S.; Dolecek, L.; Barry, J.; Sala, F.; Vasić, B. Signal processing and coding techniques for 2-D magnetic recording: An overview. Proc. IEEE. 2018, 106, 286-318. [CrossRef]

5. Rottmayer, R.E.; Batra, S.; Buechel, D.; Challener, W.A.; Hohlfeld, J.; Kubota, Y.; Li, L.; Lu, B.; Mihalcea, C.; Mountfield, K.; et al. Heat-assisted magnetic recording. IEEE Trans. Magn. 2006, 42, 2417-2421. [CrossRef]

6. Sadeghian, E.B.; Barry, J.R. Soft intertrack interference cancellation for two-dimensional magnetic recording. IEEE Trans. Magn. 2015, 51, 1-9. [CrossRef]

7. Shi, S.; Barry, J.R. Multitrack detection with 2D pattern-dependent noise prediction. In Proceedings of the 2018 IEEE International Conference on Communications (ICC), Kansas City, MO, USA, 20-24 May 2018; pp. 1-6. 
8. Cideciyan, R.D.; Dolivo, F.; Hermann, R.; Hirt, W.; Schott, W. A PRML system for digital magnetic recording. IEEE J. Sel. Areas Commun. 1992, 10, 38-56. [CrossRef]

9. Nabavi, S.; Kumar, B.V.K.V. Two-dimensional generalized partial response equalizer for bit-patterned media. In Proceedings of the 2007 IEEE International Conference on Communications, Glasgow, UK, 24-28 June 2007; pp. 6249-6254.

10. Nguyen, T.A.; Lee, J. One-dimensional serial detection using new two-dimensional partial response target modeling for bitpatterned media recording. IEEE Magn. Lett. 2020, 11, 1-5. [CrossRef]

11. Nguyen, T.A.; Lee, J. Effective generalized partial response target and serial detector for two-dimensional bit-patterned media recording channel including track mis-registration. Appl. Sci. 2020, 10, 5738. [CrossRef]

12. Kim, J.; Lee, J. Two-dimensional SOVA and LDPC codes for holographic data storage system. IEEE Trans. Magn. 2009, 45, 2260-2263.

13. Bahl, L.; Cocke, J.; Jelinek, F.; Raviv, J. Optimal decoding of linear codes for minimizing symbol error rate (Corresp.). IEEE Trans. Inform. Theory 1974, 20, 284-287. [CrossRef]

14. Jeong, S.; Kim, J.; Lee, J. Performance of bit-patterned media recording according to island patterns. IEEE Trans. Magn. 2018, 54, 1-4. [CrossRef]

15. Nguyen, C.D.; Lee, J. Scheme for utilizing the soft feedback information in bit-patterned media recording systems. IEEE Trans. Magn. 2017, 53, 1-4. [CrossRef]

16. Warisarn, C.; Arrayangkool, A.; Kovintavewat, P. An ITI-mitigating 5/6 modulation code for bit-patterned media recording. IEICE Trans. Electron. 2015, E98-C, 528-533. [CrossRef] 